\title{
Systems Biology: A Multi-Omics Integration Approach to Metabolism and the Microbiome
}

\author{
Jang Won Son ${ }^{1}$, Saeed Shoaie ${ }^{2,3}$, Sunjae Lee ${ }^{2}$ \\ ${ }^{1}$ Division of Endocrinology and Metabolism, Department of Internal Medicine, Bucheon St. Mary's Hospital, College of \\ Medicine, The Catholic University of Korea, Bucheon, Korea; ${ }^{2}$ Centre for Host-Microbiome Interactions, Faculty of Dentistry, \\ Oral \& Craniofacial Sciences, King's College London, London, UK; ${ }^{3}$ Science for Life Laboratory, KTH-Royal Institute of \\ Technology, Stockholm, Sweden
}

The complex and dynamic nature of human physiology, as exemplified by metabolism, has often been overlooked due to the lack of quantitative and systems approaches. Recently, systems biology approaches have pushed the boundaries of our current understanding of complex biochemical, physiological, and environmental interactions, enabling proactive medicine in the near future. From this perspective, we review how state-of-the-art computational modelling of human metabolism, i.e., genome-scale metabolic modelling, could be used to identify the metabolic footprints of diseases, to guide the design of personalized treatments, and to estimate the microbiome contributions to host metabolism. These state-of-the-art models can serve as a scaffold for integrating multi-omics data, thereby enabling the identification of signatures of dysregulated metabolism by systems approaches. For example, increased plasma mannose levels due to decreased uptake in the liver have been identified as a potential biomarker of early insulin resistance by multiomics approaches. In addition, we also review the emerging axis of human physiology and the human microbiome, discussing its contribution to host metabolism and quantitative approaches to study its variations in individuals.

Keywords: Systems biology; Metabolism; Gastrointestinal microbiome

\section{INTRODUCTION}

The human body maintains homeostasis through highly complex interactions of biochemical reactions, physiological processes, and environmental interactions at different scales [1]. These complex and dynamic properties have been overlooked in many disciplines of biology and medicine due to the lack of quantitative and systems approaches. Recently, many interesting strategies have been devised to simplify complex and dynamic biological problems into more understandable formulas (Fig. 1A) [2]. For example, network theory has been applied to

Received: 6 July 2020, Revised: 14 August 2020, Accepted: 19 August 2020

Corresponding author: Sunjae Lee

Centre for Host-Microbiome Interactions, Faculty of Dentistry, Oral \&

Craniofacial Sciences, King's College London, Great Maze Pond, SE1 9RT,

London, UK

Tel: +44-20-7188-9613, E-mail: sunjae.lee@kcl.ac.uk cellular regulatory networks, uncovering key driver genes or modules of disease pathogenesis [3]. Genome-scale metabolic modelling (GEM) has been employed to explain metabolic phenotypes in various living systems, including human tissues and organs, the human gut microbiome, and bacteria engineered to produce biofuels and chemicals [4-12]. In addition, computational models and networks emulating biological systems have been established and freely shared by web-based services, including Kyoto Encyclopedia of Genes and Genomes (KEGG), Reactome, Human Metabolic Atlas, and Tissue and Cancer Specific Biological Network (TCSBN) database [13-16]. These

Copyright $\odot 2020$ Korean Endocrine Society

This is an Open Access article distributed under the terms of the Creative Commons Attribution Non-Commercial License (https://creativecommons.org/ licenses/by-nc/4.0/) which permits unrestricted non-commercial use, distribution, and reproduction in any medium, provided the original work is properly cited. 

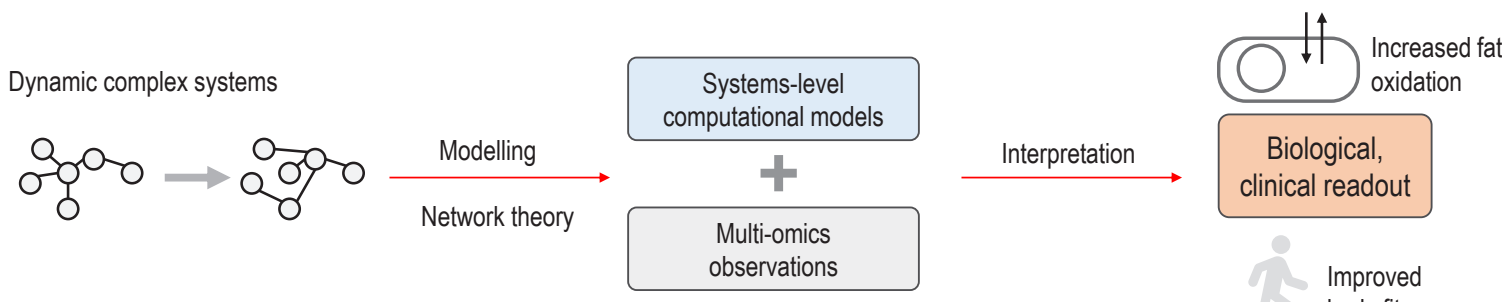

Biological, clinical readout
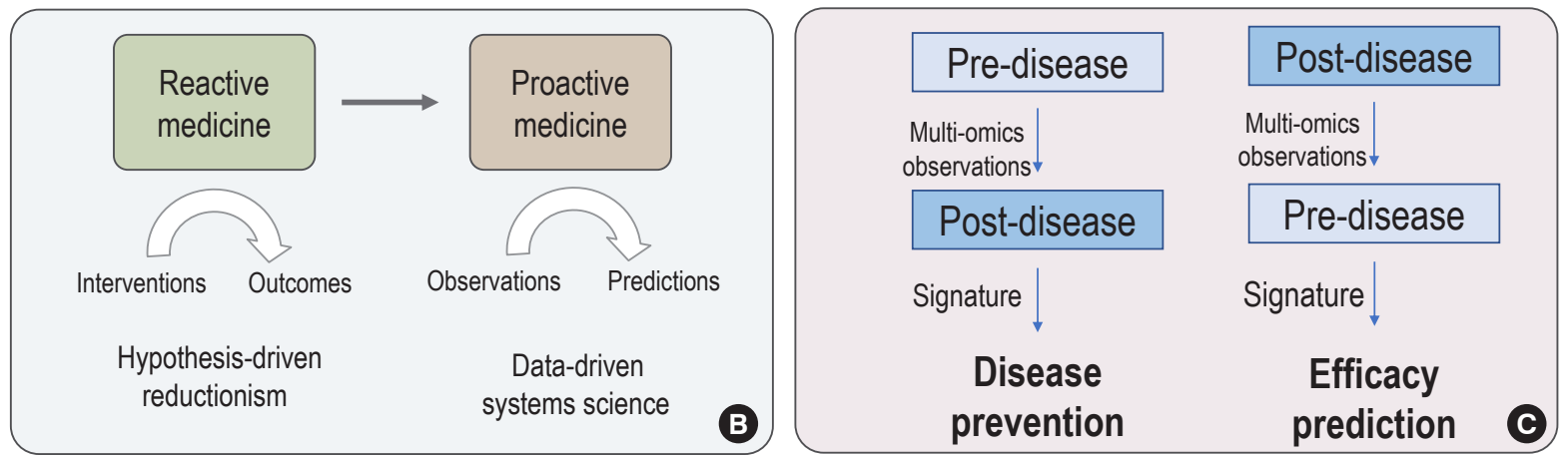

Fig. 1. Quantitative and systems approaches in biology and medicine. (A) Dynamic and complex systems can be studied with computational modelling, such as genome-scale metabolic models, and network theory, such as network biology principles. Augmenting with multi-omics observations, we could interpret complex and dynamic biological/clinical problems through more understandable readouts. (B) Based on data-driven approaches and systems science, future medicine can be transformed from reactive medicine, interpreting the outcomes of an intervention, into proactive medicine, predicting outcomes from prior observations in healthy conditions. (C) For example, the progression from pre- to post-disease states can be investigated with multi-omics datasets and its signatures could help disease prevention. Likewise, outcomes of interventions, which reverse the states from post-disease to pre-disease, can be studied with multi-omics observations, enabling the prediction of treatment efficacy.

collaborative efforts will expand our understanding of medicine, enabling proactive medicine based on personalized data clouds - that is, systems medicine (Fig. 1B).

In recent years, with the boom of high-throughput omics data, human physiology has been extensively investigated through data-driven approaches. Based on longitudinal multi-omics profiling, we can identify individual signatures of pre- and postdisease states (Fig. 1C). For example, 108 healthy individuals were monitored for a year with personal multi-omics data, including metabolomics, proteomics, and gut metagenomics [17]. Based on multi-omics observations, subjects progressing from pre-disease states, such as elevated glucose or haemoglobin A1c levels, inflammation, and iron deficiency, were coached to change their lifestyle and diet to prevent poor health outcomes. Moreover, some multi-omics studies have identified individual signatures of the transition from post-disease to pre-disease states as a result of interventions. For example, the effects of an isocaloric low-carbohydrate diet in subjects with hepatic steatosis were investigated with multi-omics data, including metabolome, biopsy (liver) transcriptome, gut metagenome, and in- flammatory markers [18]. Within 2 weeks after starting the lowcarbohydrate diet, significant reductions of liver fat, plasma triglyceride levels, and inflammatory markers were observed despite no significant weight changes. In particular, genome-scale metabolic models (GEMs) have been provided as scaffolds to explain metabolic phenotypes by integrating metabolomics and transcriptomics data. A liver GEM predicted increased fluxes through fatty acid beta-oxidation and one carbon metabolism, which generates antioxidants and cofactors, including $\mathrm{NAD}^{+}$ and glutathione, for beta-oxidation, and these increased fluxes were also linked to increased folate production of the gut microbiome.

Such advances in systems medicine would eventually allow N-of-1 trials - that is, clinical trials designed for a single patient - based on the prediction of treatment efficacy and side effects (personalized and predictive medicine). Moreover, through systems biology tools and models, possible risk factors will be prevented through active participation of customers (preventive and participatory medicine). Personalized, predictive, preventive, and participatory medicine (P4 medicine) would thereby 
transform the current form of healthcare services in the near future. Recently, international consortia of systems medicine, including the European Association of Systems Medicine (EASyM) and the Coordinating Action Systems Medicine (CASyM), have started discussions on a roadmap for implementing systems approaches into medical practice $[19,20]$, and also for systems medicine courses comprising multidisciplinary education and specific analytic skills [21].

In the following sections, we detail how systems biology methodology could help understand health and disease with concrete examples. Specifically, we explain (1) how GEM could identify tissue-specific or disease-specific metabolic signatures and new therapeutic compounds for personalized treatments; (2) how multi-omics data can be integrated into genome-scale models to identify biomarkers of dysregulated metabolism; and (3) how associations of the gut microbiome with diseases can be identified with quantitative and systems approaches.

\section{COMPUTATIONAL MAPS OF HUMAN METABOLISM: GENOME-SCALE METABOLIC MODELS}

All living organisms, including humans, survive by converting nutrients into energy and chemical substances used as the building blocks of cells [22,23]. All biochemical reactions that produce metabolites, energy, and redox power are strictly regulated in cells, especially by metabolic enzymes, and these reactions are interconnected, as different biochemical reactions share substrates. Because of the highly interconnected nature of metabolism, any changes in biological processes could affect metabolism, and therefore dysregulated processes in diseases could leave metabolic footprints [22].

Recently, the systems biology community has established a computational "map" to explore the complexity of metabolism through GEMs (Fig. 2A). In these maps, all metabolites are connected through roads ("reactions") and the traffic along all the roads are controlled by enzymes. Based on a metabolism map, we can estimate the possible degree of traffic ("flux") by linear programming (or called linear optimization), through a process known as flux balance analysis (FBA). For example, bacteria regulate metabolic fluxes to maximize the production of biomass, so we can identify higher fluxes for all the roads ("reactions") that contribute to the production of biomass by using FBA as an optimization method. However, in humans, metabolic demands vary across cells and tissues. Thus, an alternative strategy is to estimate fluxes by integrating information re- garding enzymes, which function as "traffic controllers" [4,24, $25]$. For example, when gene expression levels of glycolytic enzymes are higher in cancers, we could predict relatively higher fluxes of the corresponding enzymes.

Using this strategy, we can also generate tissue- and diseasespecific metabolic "maps," or GEMs composed of fluxes that may be maintained in given conditions (Fig. 2B) [4,25]. For example, hepatocyte, adipocyte, and myocyte GEMs were generated and analysed in the context of liver disease, obesity, and diabetes [26-28]. Using a hepatocyte GEM, serine deficiency was identified in non-alcoholic fatty liver disease (NAFLD) and elevated levels of branched amino acids in obese patients were identified using an adipocyte GEM [26,27,29].

Moreover, generating personalized GEMs could guide the treatment of individual tumors [25,30,31]. For example, dysregulated metabolites identified from personalized GEMs can be treated with anti-metabolites, which are analogue compounds of given metabolites that function as competitive inhibitors [25]. In hepatocellular carcinoma, 101 anti-metabolites have been predicted to suppress tumour growth in individuals, among which the anti-metabolite of L-carnitine, perhexiline, has had its inhibitory effect validated using the HepG2 cell line [25]. Furthermore, personalized GEMs for NAFLD have guided interventions to supplement metabolic co-factors (serine, N-acetylcysteine, nicotinamide riboside, and L-carnitine) to reduce liver fat [30,31], and its acute effects were observed based on plasma metabolomics and levels of inflammatory markers [32].

Lastly, the functional role of the human gut microbiome can be studied with GEMs. The human gut microbiome can significantly contribute to host metabolism by breaking down resistant fibres to generate short-chain fatty acids (SCFAs), such as acetate, propionate, and butyrate, and synthesizing essential amino acids and vitamins [33]. Its contribution to host metabolism can be studied with tissue-specific GEMs. For example, 28 tissuespecific GEMs from a mouse model were generated using proteomics data and the contribution of the microbiome to host metabolism was investigated. In short, by comparing gene expression levels between conventionally raised and germ-free mice, tissue GEMs predicted that host amino acid and glutathione metabolism would be different in conventionally raised mice, compared to germ-free mice [6,7]. Moreover, diet-induced metabolic changes caused by the microbiome can be studied with the state-of-the art algorithms of microbial community simulations using microbial GEMs, known as the Community And Systems-level INteractive Optimization (CASINO) toolbox [7]. Based on this microbial community algorithm, alterations of 
Genome-scale metabolic models
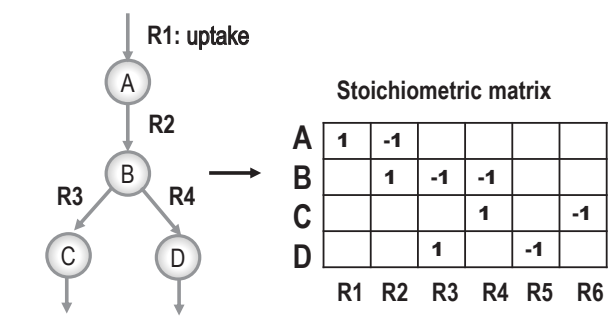

$\begin{array}{llllll}\text { R1 } & \text { R2 } & \text { R3 } & \text { R4 } & \text { R5 } & \text { R6 }\end{array}$

R5: biomass R6: secretion

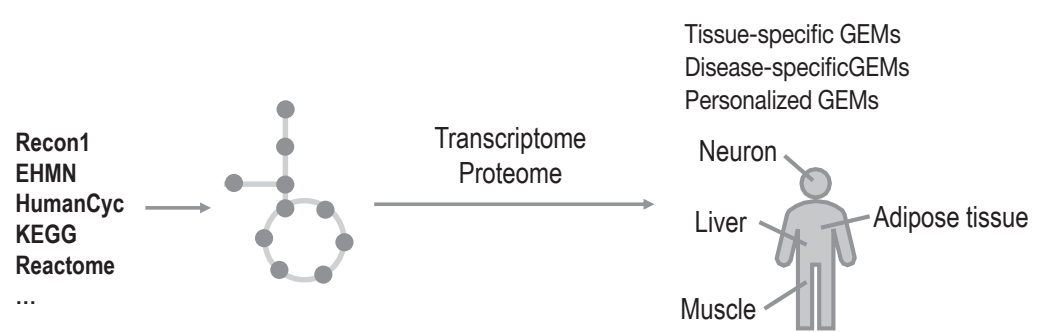

Generic human GEM
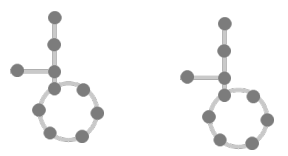

Liver GEM

HCC GEM

Fig. 2. Overview of genome-scale metabolic models (GEMs). (A) GEMs provide a computational "map" of all possible biochemical reactions within a living system, including bacteria, cells, and tissues. All biochemical reactions are composed of metabolites (e.g., metabolites from A to D) and reactions (e.g., reactions from R1 to R6) that catalyse the formation of those metabolites. In this computational map, reactions can be likened to "roads" that lead between "metabolite" destinies. Enzymes perform "traffic control" on each corresponding road, depending on cellular demands. After composing stoichiometric matrices of metabolites and reactions into GEMs, we can simulate and predict metabolic flux by linear optimization and statistical algorithms, including flux balance analysis (FBA), flux variability analysis (FVA), reporter metabolite analysis, and optimal knockout simulation. (B) A generic GEM of human metabolism has been constructed by integrating different sources of biochemical reaction knowledge, including Recon1, Edinburgh Human Metabolic Network (EHMN), HumanCyc, Kyoto Encyclopedia of Genes and Genomes (KEGG), and Reactome. The generic human GEM can be reconstructed into tissue-specific, disease-specific, or personalized GEMs based on contextual information about enzymes, including transcriptome and proteome findings. For example, liver tissue and hepatocellular carcinoma (HCC) GEMs were generated and used to identify metabolic alterations in HCC and other liver diseases and to guide the design of anti-metabolites for personalized treatments.

faecal and serum amino acid and SCFA levels were predicted and validated with faecal and blood metabolomics. In addition, personalized nutrition can be recommended based on the gut microbiome composition and diversity measure.

\section{MULTI-OMICS INTEGRATION APPROACHES}

Following the information flow in the central dogma of molecular biology, any genomic, transcriptomic, and proteomic changes can be read out in the form of metabolomic changes (Fig. 3). Therefore, multi-omics observations would yield clear insights regarding which levels of omics initiate the downstream changes to metabolic alterations in chronic diseases. Currently, thousands of risk loci of diseases have been identified by genomewide association studies (GWAS), but many of their linkages to pathophysiology have remained obscure. Recent studies have attempted to integrate GWAS findings with those of other omics studies, including RNA-seq, and by doing so have gained better insights into the identification of causal variations [34]. Likewise, multi-omics observations could identify how genetic, epigenetic, or transcriptional changes lead to metabolic alterations in complex diseases in a comprehensive manner.

Importantly, GEMs could serve as a scaffold for integrating biological networks and multi-omics datasets, thereby allowing us to trace the alterations of biomolecule abundance levels and their interactions in different omics layers. For example, by integrating tissue-specific biological networks into GEMs, such as hepatocyte, adipocyte, and myocyte GEMs, tissue-specific metabolism could be identified based on co-regulation of the corresponding enzymes. In a recent study, co-regulation analyses of integrated networks identified that cholesterol biosynthesis was highly co-regulated in the liver and interestingly, that mannose metabolism was highly co-regulated in hepatocytes, adipocytes, and myocytes [35]. Mapping the transcriptome of liver and adipose tissue biopsies, co-regulation analyses identi- 
Information flows

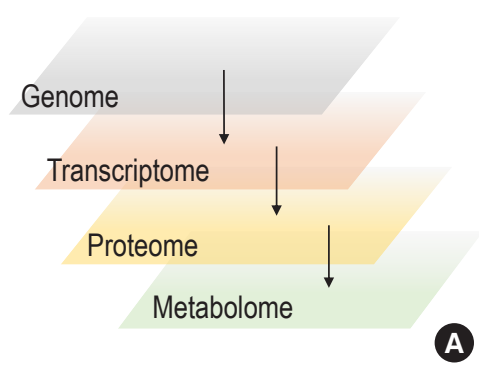

Integrated networks

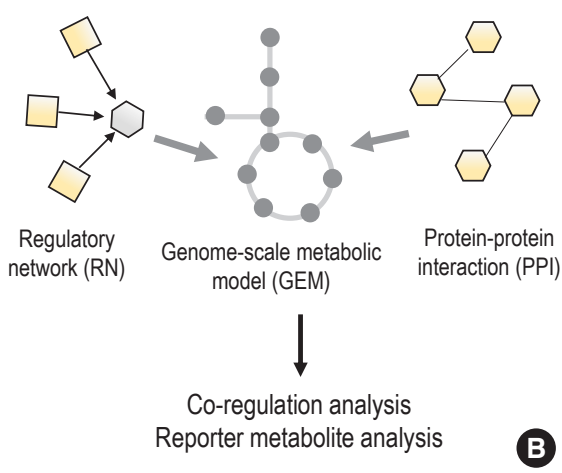

Less consumption of mannose in liver among obese subjects

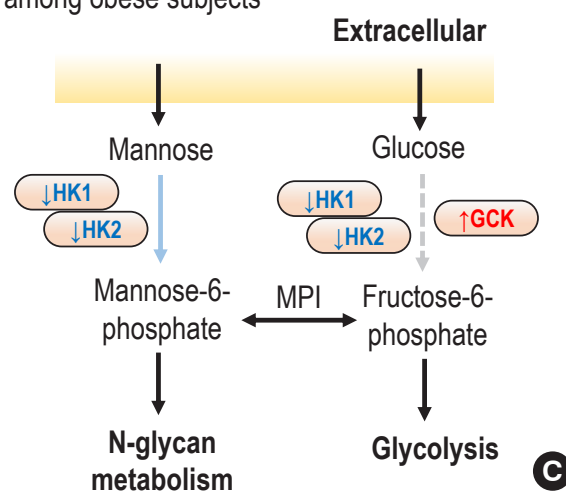

Fig. 3. Multi-omics integration approaches. (A) Based on the central dogma, information flows could affect different omics from the upstream level (the genome) to the downstream level (the metabolome). All possible changes in upstream omics can be read out into metabolomic changes. (B) In order to study possible abundance changes in different omics and changes in interactions in individuals with diseases, diverse biological networks can be integrated into genome-scale metabolic models and metabolomics changes can be interpreted by investigating integrated networks and multi-omics datasets. Co-regulation analysis could identify the densest modules that regulate specific metabolic pathways, as well as their dysregulation in disease conditions. (C) Based on a co-regulation analysis of integrated networks, and together with a reporter metabolite analysis, less consumption of mannose in liver tissue was identified among obese subjects. For example, the gene expression levels of 2 hexokinases were decreased, whereas glucokinase, the more efficient isozyme of hexokinase for glucose, showed increased gene expression among obese subjects. Therefore, we could conclude that metabolic adaptations led to increased uptake of glucose and decreased uptake of mannose in liver tissue. Interestingly, decreased levels of mannose, a major building block of glycosylation, can affect the glycosylation of hepatic insulin receptors, thereby leading to less clearance of free insulin and eventually to insulin resistance. HK, hexokinase; GCK, glucokinase; MPI, mannose phosphate isomerase.

fied that mannose metabolism in liver tissue was significantly decreased through co-regulation in obese subjects. This finding was corroborated through the analysis of an independent algorithm (reporter metabolite analysis) [36], which identified significant transcriptional changes regarding mannose uptake in the liver. The findings of decreased mannose uptake in liver tissue and subsequent increased plasma mannose levels were validated in 3 independent metabolomics cohorts: (1) lean versus obese subjects (the Relationship between Insulin Sensitivity and Cardiovascular disease [RISC] study); (2) insulin-sensitive versus insulin-resistant obese subjects (the Leipzig study); and (3) high versus low insulin-secreting subjects (the Kuopio study). Interestingly, plasma mannose was significantly enriched in obese, insulin-resistant, and low insulin-secreting subjects, even more significantly than glucose. Furthermore, an independent study of mannose supplementation suggested that mannose could improve insulin tolerance and reduce weight and body fat in mice with high-fat diets if supplemented at early stages [37]. Likewise, integrative network analyses of GEMs could identify potential biomarkers of chronic diseases through multi-omics observations in a comprehensive manner.

\section{A NEW EMERGING PARADIGM: THE HUMAN MICROBIOME}

Recently, human microbiome research has been highlighted due to its therapeutic potential beyond the knowledge of lactate-producing bacteria. For example, gut microbiota have been found to (1) successfully cure multiple diseases through transplantation from donors to patients, including Clostridium difficile infection, inflammatory bowel disease, and obesity [38-40]; and (2) modulate the efficacy of host-directed drugs, even immune check-point inhibitors [41,42].

In light of increasing demands for a better understanding of the human microbiome, many approaches have been attempted to reconstruct reference genomes for the human microbiome. However, as many species were unculturable in laboratory conditions, reference genomes for many microbes could not be obtained by in vitro isolations and remained as microbial "dark matter" [43-45]. While powerful, 16S rRNA amplicon sequencing is dependent on reference genomes from isolated bacteria, making it difficult to uncover the whole bacterial population, which includes unculturable species [46]. In addition, this method could introduce biases due to variation in 16S rRNA gene 
copy numbers, and 16S rRNA sequence conservation limits the ability of this technique to distinguish closely related organisms, posing difficulties for taxonomic classifications at the species and strain levels $[46,47]$.

Recently, computational methods that enable the direct identification of reference genomes from shotgun metagenomics were devised. For example, short reads of shotgun sequencing were assembled and binned into metagenome-assembled genomes (MAGs) based on the similarity of nucleotide compositions $[48,49]$, and co-abundant gene profiling was used to identify clustered gene groups and assemble them into metagenomics species (MGSs), or a metagenomic species pan-genome (MSP) [50,51]. These methods allow us to uncover unculturable species from MAG or MGS/MSP and thereby to quantify the microbiome composition without reference genomes known.

Based on quantitative microbiome profiling of shotgun metagenomics using isolate genomes or MGS, variations of the human microbiome have been associated with diets, diseases, and levels of drug efficacy [52]. For example, a metagenomewide association study was conducted to investigate the microbial species that are enriched or depleted in adults with diabetes [53], and functional analyses identified that elevated levels of branched amino acids in diabetes were associated with microbial dysbiosis [52]. Unsupervised clustering of human gut microbiome compositions revealed that all individuals can be classified as having three different types of microbiome, called enterotypes, which are associated with different dietary patterns [54]. For instance, the individual gut microbiome can be enriched with Bacteroides, Prevotella, or Firmicutes. Individuals with a high-fat diet and low-grade inflammation were enriched with the Bacteroides type, whereas those with a high-fibre diet were enriched with the Prevotella type. Likewise, the individual microbiome could indicate susceptibility to chronic diseases and predict drug efficacy [41,55], and even could be used in therapeutics to improve metabolic phenotypes, such as obesity [40]. Based on diet-microbiome-host interactions, microbiome studies could guide personalized nutrition for the prevention and early intervention of diseases [56].

\section{CONCLUSIONS}

The complex and dynamic nature of human physiology can be studied with systems biology approaches, simplifying complex biological problems into more understandable formulas. Based on quantitative and systems approaches, current healthcare services can be transformed to proactive medicine in the near fu- ture. For example, state-of-the-art modelling of human metabolism through GEMs could accelerate the systems understanding of metabolic alterations in diseases by providing computational "maps," enabling personalized treatments and diets to be suggested. In addition, multi-omics studies with integrative network analyses could also identify which level of omics changes eventually leads to metabolic alterations. For example, metabolic adaptations driving lower levels of mannose consumption in the liver, and subsequently increased plasma mannose levels, were identified. This lack of mannose might lead to insulin resistance because poorly glycosylated hepatic insulin receptors could not clear free insulin in the blood. As a recently emerged axis of human physiology, the human microbiome could explain unexplored variations in human physiology, and quantitative and systems approaches have been devised to study those variations in health and disease.

\section{CONFLICTS OF INTEREST}

No potential conflict of interest relevant to this article was reported.

\section{ACKNOWLEDGMENTS}

This study was funded by the Engineering and Physical Sciences Research Council (EPSRC) and Biotechnology Biological Sciences Research Council (BBSRC) EP/S001301/1 and by the Institute of Clinical Medicine Research of Bucheon St. Mary's Hospital, Research Fund, BCMC19LH03 and the Catholic Medical Center Research Foundation made in the program year of 2020 .

\section{ORCID}

Jang Won Son https://orcid.org/0000-0003-0339-0131

Sunjae Lee https://orcid.org/0000-0002-6428-5936

\section{REFERENCES}

1. Betts JG, Desaix P, Johnson E, Johnson JE, Korol O, Kruse $\mathrm{D}$, et al. Anatomy and physiology: part 2. Houston: 12th Media Services; 2016.

2. Lavrik IN, Zhivotovsky B. Systems biology: a way to make complex problems more understandable. Cell Death Dis 2014;5:e1256.

3. Barabasi AL, Gulbahce N, Loscalzo J. Network medicine: a 
network-based approach to human disease. Nat Rev Genet 2011;12:56-68.

4. Agren R, Bordel S, Mardinoglu A, Pornputtapong N, Nookaew I, Nielsen J. Reconstruction of genome-scale active metabolic networks for 69 human cell types and 16 cancer types using INIT. PLoS Comput Biol 2012;8:e1002518.

5. Mardinoglu A, Nielsen J. New paradigms for metabolic modeling of human cells. Curr Opin Biotechnol 2015;34: 91-7.

6. Shoaie S, Karlsson F, Mardinoglu A, Nookaew I, Bordel S, Nielsen J. Understanding the interactions between bacteria in the human gut through metabolic modeling. Sci Rep 2013;3:2532.

7. Shoaie S, Ghaffari P, Kovatcheva-Datchary P, Mardinoglu A, Sen P, Pujos-Guillot E, et al. Quantifying diet-induced metabolic changes of the human gut microbiome. Cell Metab 2015;22:320-31.

8. Bidkhori G, Benfeitas R, Klevstig M, Zhang C, Nielsen J, Uhlen M, et al. Metabolic network-based stratification of hepatocellular carcinoma reveals three distinct tumor subtypes. Proc Natl Acad Sci U S A 2018;115:E11874-83.

9. Benfeitas R, Bidkhori G, Mukhopadhyay B, Klevstig M, Arif $\mathrm{M}$, Zhang $\mathrm{C}$, et al. Characterization of heterogeneous redox responses in hepatocellular carcinoma patients using network analysis. EBioMedicine 2019;40:471-87.

10. Nilsson A, Mardinoglu A, Nielsen J. Predicting growth of the healthy infant using a genome scale metabolic model. NPJ Syst Biol Appl 2017;3:3.

11. Omenetti S, Bussi C, Metidji A, Iseppon A, Lee S, Tolaini M, et al. The intestine harbors functionally distinct homeostatic tissue-resident and inflammatory Th17 cells. Immunity 2019;51:77-89.

12. Liu L, Agren R, Bordel S, Nielsen J. Use of genome-scale metabolic models for understanding microbial physiology. FEBS Lett 2010;584:2556-64.

13. Kanehisa M, Furumichi M, Tanabe M, Sato Y, Morishima K. KEGG: new perspectives on genomes, pathways, diseases and drugs. Nucleic Acids Res 2017;45:D353-61.

14. Robinson JL, Kocabas P, Wang H, Cholley PE, Cook D, Nilsson A, et al. An atlas of human metabolism. Sci Signal 2020;13:eaaz1482.

15. Jassal B, Matthews L, Viteri G, Gong C, Lorente P, Fabregat A, et al. The reactome pathway knowledgebase. Nucleic Acids Res 2020;48:D498-503.

16. Lee S, Zhang C, Arif M, Liu Z, Benfeitas R, Bidkhori G, et al. TCSBN: a database of tissue and cancer specific biologi- cal networks. Nucleic Acids Res 2018;46:D595-600.

17. Price ND, Magis AT, Earls JC, Glusman G, Levy R, Lausted $\mathrm{C}$, et al. A wellness study of 108 individuals using personal, dense, dynamic data clouds. Nat Biotechnol 2017;35:747-56.

18. Mardinoglu A, Wu H, Bjornson E, Zhang C, Hakkarainen A, Rasanen SM, et al. An integrated understanding of the rapid metabolic benefits of a carbohydrate-restricted diet on hepatic steatosis in humans. Cell Metab 2018;27:559-71.

19. Kirschner M, Bauch A, Agusti A, Hilke S, Merk S, Pison C, et al. Implementing systems medicine within healthcare. Genome Med 2015;7:102.

20. Auffray C. Interview with a thought leader on systems medicine. Syst Med 2018;1:11-2.

21. Gomez-Cabrero D, Marabita F, Tarazona S, Cano I, Roca J, Conesa A, et al. Guidelines for developing successful short advanced courses in systems medicine and systems biology. Cell Syst 2017;5:168-75.

22. Nielsen J. Systems biology of metabolism: a driver for developing personalized and precision medicine. Cell Metab 2017;25:572-9.

23. Nielsen J. Systems biology of metabolism. Annu Rev Biochem 2017;86:245-75.

24. O'Brien EJ, Monk JM, Palsson BO. Using genome-scale models to predict biological capabilities. Cell 2015;161:97187.

25. Agren R, Mardinoglu A, Asplund A, Kampf C, Uhlen M, Nielsen J. Identification of anticancer drugs for hepatocellular carcinoma through personalized genome-scale metabolic modeling. Mol Syst Biol 2014;10:721.

26. Mardinoglu A, Agren R, Kampf C, Asplund A, Nookaew I, Jacobson $\mathrm{P}$, et al. Integration of clinical data with a genomescale metabolic model of the human adipocyte. Mol Syst Biol 2013;9:649.

27. Mardinoglu A, Agren R, Kampf C, Asplund A, Uhlen M, Nielsen J. Genome-scale metabolic modelling of hepatocytes reveals serine deficiency in patients with non-alcoholic fatty liver disease. Nat Commun 2014;5:3083.

28. Varemo L, Scheele C, Broholm C, Mardinoglu A, Kampf C, Asplund A, et al. Proteome- and transcriptome-driven reconstruction of the human myocyte metabolic network and its use for identification of markers for diabetes. Cell Rep 2015;11:921-33.

29. Mardinoglu A, Kampf C, Asplund A, Fagerberg L, Hallstrom BM, Edlund K, et al. Defining the human adipose tissue proteome to reveal metabolic alterations in obesity. J Proteome Res 2014;13:5106-19. 
30. Mardinoglu A, Bjornson E, Zhang C, Klevstig M, Soderlund S, Stahlman M, et al. Personal model-assisted identification of NAD + and glutathione metabolism as intervention target in NAFLD. Mol Syst Biol 2017;13:916.

31. Mardinoglu A, Boren J, Smith U, Uhlen M, Nielsen J. Systems biology in hepatology: approaches and applications. Nat Rev Gastroenterol Hepatol 2018;15:365-77.

32. Zhang C, Bjornson E, Arif M, Tebani A, Lovric A, Benfeitas $\mathrm{R}$, et al. The acute effect of metabolic cofactor supplementation: a potential therapeutic strategy against non-alcoholic fatty liver disease. Mol Syst Biol 2020;16:e9495.

33. Koh A, De Vadder F, Kovatcheva-Datchary P, Backhed F. From dietary fiber to host physiology: short-chain fatty acids as key bacterial metabolites. Cell 2016;165:1332-45.

34. Karczewski KJ, Snyder MP. Integrative omics for health and disease. Nat Rev Genet 2018;19:299-310.

35. Lee S, Zhang C, Kilicarslan M, Piening BD, Bjornson E, Hallstrom BM, et al. Integrated network analysis reveals an association between plasma mannose levels and insulin resistance. Cell Metab 2016;24:172-84.

36. Patil KR, Nielsen J. Uncovering transcriptional regulation of metabolism by using metabolic network topology. Proc Natl Acad Sci U S A 2005;102:2685-9.

37. Sharma V, Smolin J, Nayak J, Ayala JE, Scott DA, Peterson $\mathrm{SN}$, et al. Mannose alters gut microbiome, prevents diet-induced obesity, and improves host metabolism. Cell Rep 2018;24:3087-98.

38. Kao D, Roach B, Silva M, Beck P, Rioux K, Kaplan GG, et al. Effect of oral capsule- vs colonoscopy-delivered fecal microbiota transplantation on recurrent clostridium difficile infection: a randomized clinical trial. JAMA 2017;318: 1985-93.

39. Khoruts A, Sadowsky MJ. Understanding the mechanisms of faecal microbiota transplantation. Nat Rev Gastroenterol Hepatol 2016;13:508-16.

40. Depommier C, Everard A, Druart C, Plovier H, Van Hul M, Vieira-Silva S, et al. Supplementation with Akkermansia muciniphila in overweight and obese human volunteers: a proofof-concept exploratory study. Nat Med 2019;25:1096-103.

41. Gopalakrishnan V, Spencer CN, Nezi L, Reuben A, Andrews MC, Karpinets TV, et al. Gut microbiome modulates response to anti-PD-1 immunotherapy in melanoma patients. Science 2018;359:97-103.

42. Koppel N, Maini Rekdal V, Balskus EP. Chemical transformation of xenobiotics by the human gut microbiota. Science 2017;356:eaag2770.
43. Dance A. The search for microbial dark matter. Nature 2020; 582:301-3.

44. Lagier JC, Dubourg G, Million M, Cadoret F, Bilen M, Fenollar F, et al. Culturing the human microbiota and culturomics. Nat Rev Microbiol 2018;16:540-50.

45. Lok C. Mining the microbial dark matter. Nature 2015;522: 270-3.

46. Milanese A, Mende DR, Paoli L, Salazar G, Ruscheweyh HJ, Cuenca M, et al. Microbial abundance, activity and population genomic profiling with mOTUs2. Nat Commun 2019;10:1014.

47. Hillmann B, Al-Ghalith GA, Shields-Cutler RR, Zhu Q, Gohl DM, Beckman KB, et al. Evaluating the information content of shallow shotgun metagenomics. mSystems 2018; 3:e00069-18.

48. Nayfach S, Shi ZJ, Seshadri R, Pollard KS, Kyrpides NC. New insights from uncultivated genomes of the global human gut microbiome. Nature 2019;568:505-10.

49. Kang DD, Li F, Kirton E, Thomas A, Egan R, An H, et al. MetaBAT 2: an adaptive binning algorithm for robust and efficient genome reconstruction from metagenome assemblies. PeerJ 2019;7:e7359.

50. Nielsen HB, Almeida M, Juncker AS, Rasmussen S, Li J, Sunagawa $\mathrm{S}$, et al. Identification and assembly of genomes and genetic elements in complex metagenomic samples without using reference genomes. Nat Biotechnol 2014;32: 822-8.

51. Plaza Onate F, Le Chatelier E, Almeida M, Cervino ACL, Gauthier F, Magoules F, et al. MSPminer: abundance-based reconstitution of microbial pan-genomes from shotgun metagenomic data. Bioinformatics 2019;35:1544-52.

52. Wang J, Jia H. Metagenome-wide association studies: finemining the microbiome. Nat Rev Microbiol 2016;14:50822.

53. Qin J, Li Y, Cai Z, Li S, Zhu J, Zhang F, et al. A metagenome-wide association study of gut microbiota in type 2 diabetes. Nature 2012;490:55-60.

54. Arumugam M, Raes J, Pelletier E, Le Paslier D, Yamada T, Mende DR, et al. Enterotypes of the human gut microbiome. Nature 2011;473:174-80.

55. Chiu CY, Miller SA. Clinical metagenomics. Nat Rev Genet 2019;20:341-55.

56. Zeevi D, Korem T, Zmora N, Israeli D, Rothschild D, Weinberger A, et al. Personalized nutrition by prediction of glycemic responses. Cell 2015;163:1079-94. 